\title{
EXACT QUANTUM STATES FOR ALL TWO-DIMENSIONAL DILATON GRAVITY THEORIES
}

\author{
by \\ Domingo Louis-Martinez \\ Department of Physics, University of British Columbia \\ Vancouver, BC, Canada, V6T $1 Z 1$ \\ [e-mail: martinez@physics.ubc.ca]
}

\begin{abstract}
It is shown that the recently obtained quantum wave functionals in terms of the CJZ variables for generic $2 \mathrm{~d}$ dilaton gravity are equivalent to the previously reported exact

quantum wave functionals in geometrical variables. A third representation of these exact quantum states is also presented.
\end{abstract}




\section{Introduction}

Two dimensional (2d) theories of gravity have attracted a lot of attention in recent years as a simplified theoretical model for studying Hawking evaporation of black holes and the information loss problem [1]. It is believed that these theories could provide some insight into the problems that appear in the quantization of Einstein's theory of gravity [2].

The exact quantum wave functionals for generic 2 d dilaton gravity were obtained in [3]. They were given as functionals of the spatial component $g_{11}$ of the metric and the dilaton field $\phi$ as,

$$
\Psi\left[C ; g_{11}, \phi\right]=\chi[C] \exp \left[\frac{i}{\hbar} \int_{x_{-}}^{x_{+}} d x\left(Q+\phi^{\prime} \ln \left(\frac{2 \phi^{\prime}-Q}{2 \phi^{\prime}+Q}\right)\right)\right]
$$

where $C$ is a parameter, $\chi$ is any function of $C$, and,

$$
Q=2 \sqrt{\left(\phi^{\prime}\right)^{2}+(C-j(\phi)) g_{11}}
$$

The two dimensional spacetime is assumed to be locally a direct product $R \times \Sigma$, where the spatial manifold $\Sigma$ can be either open or closed. The spatial coordinate $x$ runs from $x_{-}$to $x_{+}$.

Notice that for any $2 \mathrm{~d}$ dilaton gravity theory the action can always be written as [3:

$$
S=\int d^{2} x \sqrt{-g}(\phi R+V(\phi))
$$

where $R$ is the scalar curvature.

For spherically symmetric gravity [4] $V=\Lambda \phi^{-\frac{1}{2}}$, for the Jackiw-Teitelboim model [5] $V=\Lambda \phi$ and for the string inspired model [6, 7] $V=\Lambda$. Two-dimensional dilaton gravity models with nonsingular black hole solutions have also been found [8].

The functions $V$ and $j$ are related as follows: 


$$
\frac{d j(\phi)}{d \phi}=V(\phi)
$$

A gauge theoretical formulation for string-inspired gravity was developed in [7]. In [9] it was proven that the quantum wave functionals obtained in [7] are equivalent to the ones of [3] (for string inspired model $j=\Lambda \phi$ ).

Recently, Barvinsky and Kunstatter [10] generalized the work of [2] and found an exact expression for the quantum wave functionals for all $2 \mathrm{~d}$ dilaton gravity theories (3) in the CJZ [2] variables:

$$
\Psi\left[C ; \eta^{0}, \eta^{1}\right]=\chi[C] \exp \left[\frac{i}{\hbar} \int_{x_{-}}^{x_{+}} d x \omega\left(\eta^{2}\right)\left(\eta^{0}\left(\eta^{1}\right)^{\prime}-\eta^{1}\left(\eta^{0}\right)^{\prime}\right)\right] \exp \left(\frac{i}{\hbar} \alpha \beta\right)
$$

where the function $\omega$ is defined by the equation:

$$
C-j\left(\frac{1}{2} \eta^{2} \omega\left(\eta^{2}\right)\right)=\frac{\eta^{2}}{4}
$$

$\alpha$ is a constant (in [10], due to boundary conditions, $\alpha$ was chosen to be equal to $\left.j^{-1}(C)\right)$ and,

$$
\beta=\left.2 \tanh ^{-1}\left(\frac{\eta^{0}}{\eta^{1}}\right)\right|_{x_{-}} ^{x_{+}}
$$

Notice that,

$$
\eta^{2} \equiv\left(\eta^{1}\right)^{2}-\left(\eta^{0}\right)^{2}
$$

As can be seen from (11) or (5), for all 2d dilaton gravity theories the exact solutions are also the lowest order WKB solutions.

The purpose of this paper is to provide a proof of the equivalence of (11) and (5). A third representation for the exact quantum states in terms of the dilaton field and the momentum conjugate to the spatial component of the metric is also presented. 


\section{Proof of the equivalence}

Assume the classical phase space variables $(q, p)$ and $(Q, P)$ are related by a canonical transformation generated by the function $F(q, Q)$ :

$$
p=\frac{\partial F}{\partial q} \quad P=-\frac{\partial F}{\partial Q}
$$

where,

$$
\frac{\partial^{2} F}{\partial q \partial Q} \neq 0
$$

Assume that the eigenvectors of the operators $\hat{q}$ form a basis and that the eigen-

vectors of $\hat{Q}$ also form a basis 円. A vector $\mid \psi>$ can be defined by its wave function $\psi(q)$ in one basis or by its wave function $\Psi(Q)$ in the other.

Notice that if $\psi(q)$ is determined (up to a multiplicative constant) by the equations:

$$
-i \hbar \frac{\partial}{\partial q} \psi(q)=p(q) \psi(q)
$$

and $\Psi(Q)$ by the equations:

$$
-i \hbar \frac{\partial}{\partial Q} \Psi(Q)=P(Q) \Psi(Q)
$$

where $P(Q)$ in (12) is given by,

$$
P(Q)=-\left.\frac{\partial F}{\partial Q}(q, Q)\right|_{q=q(Q)}
$$

and $q=q(Q)$ are the solutions of the equations:

$$
p(q)-\frac{\partial F}{\partial q}(q, Q)=0
$$

\footnotetext{
${ }^{1}$ For canonical transformations of a certain type 11 the transformation function relating the two bases is given by [1]: $\exp \left(-\frac{i}{\hbar} F(q, Q)\right)$.
} 
then,

$$
\Psi(Q)=\left.\left(e^{-\frac{i}{\hbar} F(q, Q)} \psi(q)\right)\right|_{q=q(Q)}
$$

Indeed, it is easy to see that (15) satisfies (12).

The quantum wave functional (11) is the solution of the equations [3]:

$$
\begin{aligned}
& -i \hbar \frac{\delta}{\delta \phi} \Psi[C ; \rho, \phi]=\frac{g[\rho, \phi]}{Q[C ; \rho, \phi]} \Psi[C ; \rho, \phi] \\
& -i \hbar \frac{\delta}{\delta \rho} \Psi[C ; \rho, \phi]=Q[C ; \rho, \phi] \Psi[C ; \rho, \phi]
\end{aligned}
$$

where,

$$
\rho=\frac{1}{2} \ln \left(g_{11}\right)
$$

and the functions $g$ and $Q$ are,

$$
\begin{gathered}
g[\rho, \phi]=4 \phi^{\prime \prime}-4 \phi^{\prime} \rho^{\prime}-2 V(\phi) e^{2 \rho} \\
Q[C ; \rho, \phi]=2 \sqrt{\left(\phi^{\prime}\right)^{2}+(C-j(\phi)) e^{2 \rho}}
\end{gathered}
$$

The canonical transformation relating the geometrical phase space variables $\left(\rho, \phi, \Pi_{\rho}, \Pi_{\phi}\right)$ and the CJZ variables $\left(\eta^{0}, \eta^{1}, P_{0}, P_{1}\right)$ is defined as follows [2]:

$$
\begin{gathered}
e^{2 \rho}=P^{2} \equiv P_{1}^{2}-P_{0}^{2} \\
\phi^{\prime}=\frac{1}{2}\left(\eta^{0} P_{1}+\eta^{1} P_{0}\right) \\
\Pi_{\rho}=-\eta^{0} P_{0}-\eta^{1} P_{1}
\end{gathered}
$$




$$
\Pi_{\phi}=\frac{2}{P^{2}}\left(P_{0} P_{1}^{\prime}-P_{1} P_{0}^{\prime}\right)
$$

The generating functional $F\left[\rho, \phi ; \eta^{0}, \eta^{1}\right]$ can be written as,

$$
\begin{gathered}
F\left[\rho, \phi ; \eta^{0}, \eta^{1}\right]=\int_{x_{-}}^{x_{+}} d x\left[2 \sqrt{\left(\phi^{\prime}\right)^{2}+\frac{\eta^{2}}{4} e^{2 \rho}}+\phi^{\prime} \ln \left(\frac{\phi^{\prime}-\sqrt{\left(\phi^{\prime}\right)^{2}+\frac{\eta^{2}}{4} e^{2 \rho}}}{\phi^{\prime}+\sqrt{\left(\phi^{\prime}\right)^{2}+\frac{\eta^{2}}{4} e^{2 \rho}}}\right)-\right. \\
\left.-\frac{2(\phi-\alpha)}{\eta^{2}}\left(\eta^{0}\left(\eta^{1}\right)^{\prime}-\eta^{1}\left(\eta^{0}\right)^{\prime}\right)\right]
\end{gathered}
$$

where $\alpha$ is a constant.

Notice that from (25) it follows that:

$$
\begin{aligned}
& \frac{\delta F}{\delta \rho}=2 \sqrt{\left(\phi^{\prime}\right)^{2}+\frac{\eta^{2}}{4} e^{2 \rho}} \\
& \frac{\delta F}{\delta \phi}=\frac{2\left(\phi^{\prime \prime}-\phi^{\prime} \rho^{\prime}\right)-\phi^{\prime}\left(\ln \eta^{2}\right)^{\prime}}{\sqrt{\left(\phi^{\prime}\right)^{2}+\frac{\eta^{2}}{4} e^{2 \rho}}}-\frac{2}{\eta^{2}}\left(\eta^{0}\left(\eta^{1}\right)^{\prime}-\eta^{1}\left(\eta^{0}\right)^{\prime}\right)
\end{aligned}
$$

For the particular system that we are studying Eqs (14) take the form,

$$
\begin{gathered}
Q[C ; \rho, \phi]-\frac{\delta F}{\delta \rho}\left(\rho, \phi ; \eta^{0}, \eta^{1}\right)=0 \\
\frac{g[\rho, \phi]}{Q[C ; \rho, \phi]}-\frac{\delta F}{\delta \phi}\left(\rho, \phi ; \eta^{0}, \eta^{1}\right)=0
\end{gathered}
$$

From these equations we can find $\phi$ and $\rho$ as functionals of $\eta^{0}$ and $\eta^{1}$. In particular we find that,

$$
C-j(\phi)=\frac{\eta^{2}}{4}
$$

Consider now the right hand side of Eq (15). From (25) and (1) it follows that:

$$
\left.\left(e^{-\frac{i}{\hbar} F\left[\rho, \phi ; \eta^{0}, \eta^{1}\right]} \Psi[C ; \rho, \phi]\right)\right|_{C-j(\phi)=\frac{\eta^{2}}{4}}=\exp \left[\frac{i}{\hbar} \int_{x_{-}}^{x_{+}} d x \frac{2\left(\phi\left(\eta^{2}\right)-\alpha\right)}{\eta^{2}}\left(\eta^{0}\left(\eta^{1}\right)^{\prime}-\eta^{1}\left(\eta^{0}\right)^{\prime}\right)\right]
$$


where, $\phi=\phi\left(\eta^{2}\right)$ is the solution of the equation (30).

Define the function $\omega$ in the following way:

$$
\phi\left(\eta^{2}\right) \equiv \frac{1}{2} \eta^{2} \omega\left(\eta^{2}\right)
$$

Substituting (32) into (31) we finally obtain,

$$
\Psi\left[C ; \eta^{0}, \eta^{1}\right]=\chi[C] \exp \left[\frac{i}{\hbar} \int_{x_{-}}^{x_{+}} d x \omega\left(\eta^{2}\right)\left(\eta^{0}\left(\eta^{1}\right)^{\prime}-\eta^{1}\left(\eta^{0}\right)^{\prime}\right)\right] \exp \left(\frac{i}{\hbar} \alpha \beta\right)
$$

where,

$$
C-j\left(\frac{1}{2} \eta^{2} \omega\left(\eta^{2}\right)\right)=\frac{\eta^{2}}{4}
$$

and,

$$
\beta \equiv-2 \int_{x_{-}}^{x_{+}} d x \frac{\left(\eta^{0}\left(\eta^{1}\right)^{\prime}-\eta^{1}\left(\eta^{0}\right)^{\prime}\right)}{\eta^{2}}=\left.2 \tanh ^{-1}\left(\frac{\eta^{0}}{\eta^{1}}\right)\right|_{x_{-}} ^{x_{+}}
$$

Therefore, we have obtained (5) directly from (11).

Viceversa, since the wave functional in terms of the CJZ variables (5) is the solution of the equations:

$$
\begin{aligned}
-i \hbar \frac{\delta}{\delta \eta^{0}} \Psi\left[C ; \eta^{0}, \eta^{1}\right] & =2\left(\omega+\eta^{2} \frac{\partial \omega}{\partial \eta^{2}}\right)\left(\eta^{1}\right)^{\prime} \Psi\left[C ; \eta^{0}, \eta^{1}\right] \\
-i \hbar \frac{\delta}{\delta \eta^{1}} \Psi\left[C ; \eta^{0}, \eta^{1}\right] & =-2\left(\omega+\eta^{2} \frac{\partial \omega}{\partial \eta^{2}}\right)\left(\eta^{0}\right)^{\prime} \Psi\left[C ; \eta^{0}, \eta^{1}\right]
\end{aligned}
$$

we can use the same procedure to obtain (1) from (5).

This concludes the proof of the equivalence. We can view (11) and (5) as two equivalent wave functionals representing the exact quantum state $\mid \Psi_{C}>$. 


\section{A third representation for the exact quantum states $\mid \Psi_{C}>$}

A third representation for the exact quantum states $\mid \Psi_{C}>$ can be obtained by following a similar procedure as the one used in [3].

Notice that from the action (3) the following secondary first-class constraints can be obtained [3]:

$$
\begin{aligned}
\rho^{\prime} \Pi_{\rho}+\phi^{\prime} \Pi_{\phi}-\Pi_{\rho}^{\prime} & \approx 0 \\
\left(\phi^{\prime}\right)^{2}-\frac{\Pi_{\rho}^{2}}{4}+(C-j(\phi)) e^{2 \rho} & \approx 0
\end{aligned}
$$

In the quantum theory, let us define the operators

$$
\begin{aligned}
\hat{\Pi}_{\phi} & =-i \hbar \frac{\delta}{\delta \phi} \\
\hat{\rho} & =+i \hbar \frac{\delta}{\delta \Pi_{\rho}}
\end{aligned}
$$

One can check that the wave functional,

$$
\Psi\left[C ; \Pi_{\rho}, \phi\right]=\chi[C] \exp \left[\frac{i}{\hbar} \int_{x_{-}}^{x_{+}} d x\left(\Pi_{\rho}+\phi^{\prime} \ln \left(\frac{2 \phi^{\prime}-\Pi_{\rho}}{2 \phi^{\prime}+\Pi_{\rho}}\right)-\frac{\Pi_{\rho}}{2} \ln \left(\frac{\left(\phi^{\prime}\right)^{2}-\frac{\Pi_{\rho}^{2}}{4}}{j(\phi)-C}\right)\right)\right]
$$

satisfies the quantum constraints:

$$
\begin{aligned}
\left(\Pi_{\rho} \hat{\rho}^{\prime}+\phi^{\prime} \hat{\Pi}_{\phi}-\Pi_{\rho}^{\prime}\right) \Psi\left[C ; \Pi_{\rho}, \phi\right] & \equiv 0 \\
\left(\hat{\rho}-\frac{1}{2} \ln \left(\frac{\left(\phi^{\prime}\right)^{2}-\frac{\Pi_{\rho}^{2}}{4}}{j(\phi)-C}\right)\right) \Psi\left[C ; \Pi_{\rho}, \phi\right] & \equiv 0
\end{aligned}
$$

Equation (42) is indeed another representation for the exact quantum states $\mid \Psi_{C}>$. 
The equivalence of (42) with (11) and (5) can be established using the procedure presented in section 2 .

For completeness we report the generating functional $F\left[\Pi_{\rho}, \phi ; \eta^{0}, \eta^{1}\right]$ of the canonical transformation (21 - 24):

$$
\begin{aligned}
F\left[\Pi_{\rho}, \phi ; \eta^{0}, \eta^{1}\right]=\int_{x_{-}}^{x_{+}} d x\left[\frac{\Pi_{\rho}}{2}\right. & \ln \left(\frac{e^{2} \eta^{2}}{\Pi_{\rho}^{2}-4\left(\phi^{\prime}\right)^{2}}\right)+\phi^{\prime} \ln \left(\frac{2 \phi^{\prime}-\Pi_{\rho}}{2 \phi^{\prime}+\Pi_{\rho}}\right)- \\
& \left.-\frac{2(\phi-\alpha)}{\eta^{2}}\left(\eta^{0}\left(\eta^{1}\right)^{\prime}-\eta^{1}\left(\eta^{0}\right)^{\prime}\right)\right]
\end{aligned}
$$

where $e$ is the Euler number.

\section{Conclusions}

We have proven that the quantum wave functionals in the CJZ variables of [10 and the wave functionals in geometric variables of [3] are exactly equivalent. We also presented a third representation of the exact quantum states for generic $2 \mathrm{~d}$ dilaton

gravity $\mid \Psi_{C}>$ in terms of the dilaton field and the momentum conjugate to the spatial component of the metric tensor.

Construction of the quantum theory also requires defining a scalar product on the subspace spanned by the physical states $\left|\Psi_{C}\right\rangle$. I have not addressed this problem here.

The CJZ representation of the exact quantum states for 2 d gravity coupled to an abelian field can be obtained, using the approach presented here, directly from the expression given in [12].

\section{Acknowledgements}

I am very grateful to Gabor Kunstatter for letting me know the results of his paper prior to its publication and for useful conversations and comments. I would also like 
to thank Gordon Semenoff for useful conversations. This work was supported by the National Sciences and Engineering Research Council of Canada. 


\section{References}

[1] C.G. Callan, S.B. Giddings, J.A. Harvey and A. Strominger, Phys. Rev. D45, R1005 (1992). 34

[2] D. Cangemi, R. Jackiw and B. Zwiebach, Ann. Phys. (N.Y.) 245 ,408 (1996); E. Benedict, R. Jackiw and H.J. Lee, MIT preprint MIT-CTP-2544, hep-th/9607062 (1996) (to appear in Phys. Rev. D).

[3] D. Louis-Martinez, J. Gegenberg and G. Kunstatter, Phys. Letts. B321, 193 (1994).

[4] J. Gegenberg and G. Kunstatter, Phys. Rev. D47, R4192 (1993); W.G. Unruh, Phys. Rev. D14, 870 (1976); K. Kuchar, Phys. Rev. D50, 3961 (1994).

[5] R. Jackiw, in Quantum Theory of Gravity, edited by S. Christesen (Hilger, Britol, 1984), p. 403; C. Teitelboim, ibid, p. 327; M. Henneaux, Phys. Rev. Lett. 54, 959 (1985).

[6] E. Witten, Phys. Rev. D44, 314 (1991); G. Mandal, A.M. Sengupta and S.R. Nadia, Mod. Phys. Lett. A6, 1685 (1991).

[7] D. Cangemi and R. Jackiw, Phys. Rev. D50, 3913 (1994), Phys. Lett. B337, 271 (1994).

[8] M. Trodden, V.F. Mukhanov and R.H. Brandenberger, Phys. Letts. B316, 483 (1993).

[9] E. Benedict, Phys. Lett. B340, 43 (1994).

[10] A. Barvinski and G. Kunstatter, Exact Physical Black Hole States in Generic 2D Dilaton Gravity, MIT preprint MIT-CTP-2528 (1996), hep-th/9606134 (to appear in Phys. Letts. B). 
[11] P.A.M. Dirac, Proc. Roy. Soc. (London) A, 113, 621 (1927).

[12] D. Louis-Martinez and G. Kunstatter, Phys. Rev. D52, 3494 (1995). 